\title{
LOCALIZATION OF A THEOREM OF GLIMM
}

\section{JACQUES DIXMIER}

1. Introduction. For the terminology and the main results concerning $C^{*}$-algebras, we refer to [1] where the reader will find the references. Let us just recall that if $H$ is a Hilbert space, we denote by $\mathfrak{L e}(H)$ the set of all compact linear operators in $H$, and that a $C^{*}$. algebra is said to be elementary if it is isomorphic to some algebra $\mathfrak{L e}(H)$.

Glimm's theorem [5, TheOREms 1 and 2]. Let $A$ be a separable $C^{*}$-algebra. The following conditions are equivalent;

(i) $A$ is postliminar (= GCR);

(ii) $A$ is type I;

(iii) $A$ has no type II representation;

(iv) $A$ has no type III representation;

(v) for every irreducible representation $\pi$ of $A, \pi(A)$ contains $\mathfrak{L C}\left(H_{\pi}\right)\left(H_{\pi}\right.$, Hilbert space of $\left.\pi\right)$;

(vi) if $\pi_{1}, \pi_{2}$ are two irreducible representations of $A$ with the same kernel, $\pi_{1}$ and $\pi_{2}$ are (unitarily) equivalent;

(vii) the Mackey Borel structure on $\hat{A}$ is standard;

(viii) the Mackey Borel structure on $\hat{A}$ is metrically countably separated.

So, a separable $C^{*}$-algebra $A$ is either "very good" or "very bad" according as it is or it is not postliminar. We shall see that the same strong dichotomy occurs if we study the representations of $A$ with kernel a fixed primitive ideal of $A$. This will sharpen a part of Glimm's theorem.

The proof is a synthesis of known arguments.

2. Let $X_{0}$ be the group $Z / 2 Z$ with two elements 0,1 ; we shall denote by $X$ the compact metrizable group $X_{0} \times X_{0} \times X_{0} \times \cdots$, by $X^{\prime}$ the subgroup of $X$ consisting of those elements with all but a finite number of coordinates equal to 0 . Let $E$ be a Borel space. It follows from [5, p. 593, lines $1-11$ and p. 596, lines 7-8] that if there exists an injective Borel map from $X / X^{\prime}$ into $E$, then $E$ is not metrically countably separated.

3. Theorem. Let $A$ be a separable $C^{*}$-algebra, $J$ a primitive ideal of $A, E$ the set of elements in $\hat{A}$ with kernel $J$; we consider on $E$ the Mackey Borel structure.

(a) Suppose that $A / J$ has a nonzero elementary closed two-sided ideal.

Received by the editors January 2, 1967. 
Then $E$ reduces to one point $\pi$, and $\pi(A) \supset \mathfrak{L C}\left(H_{\pi}\right)$; every factor representation of $A$ with kernel $J$ is type I.

(b) Suppose that $A / J$ has no nonzero elementary closed two-sided ideal. Then $E$ has the power of the continuum; there exists an injective Borel map from $X / X^{\prime}$ into $E$; for every $\pi \in E$ one has $\pi(A) \cap \& \mathbb{e}\left(H_{x}\right)$ $=0 ; A$ has a type II factor representation with kernel $J$ and a type III factor representation with kernel $J$.

One is reduced at once to the case where $J=0$. So we shall assume that $A$ is primitive.

Suppose that $A$ has a nonzero elementary closed two-sided ideal $K$. Since $A$ is primitive, there exists a faithful irreducible representation $\pi$ of $A$. The restriction of $\pi$ to $K$ is faithful irreducible [1, 2.11.3] whence $\pi(K)=\mathfrak{L e}\left(H_{\pi}\right)[1,4.1 .5]$ so that $\pi(A) \supset \mathscr{L C}\left(H_{\pi}\right)$. Every faithful irreducible representation of $A$ is equivalent to $\pi[1,4.1 .10]$. From this and from the reasoning of $[1$, p. $168,(v) \Rightarrow$ (ii) $]$, it results that every faithful factor representation of $A$ is type $\mathrm{I}$. This proves (a).

Suppose that $A$ has no nonzero elementary closed two-sided ideal. Let $K$ be the largest postliminar closed two-sided ideal of $A$, and let $\pi \in E$. If $K \neq 0, \pi \mid K$ is irreducible $[1,2.11 .3]$, whence $\pi(K) \supset \mathfrak{L}\left(H_{\pi}\right)$ $[1,4.3 .7]$, and so $A$ contains a nonzero elementary closed two-sided ideal contrary to the hypothesis. So $K=0$, and $A$ is antiliminar (=NGCR). On the other hand, if $\pi(A) \cap \mathfrak{e}\left(H_{\pi}\right) \neq 0$, one has $\pi(A) \supset \mathfrak{L C}\left(H_{\pi}\right)[1,4.1 .10]$, which is still absurd; this proves that $\pi(A) \cap \mathfrak{L C}\left(H_{\pi}\right)=0$.

Since $A$ is separable antiliminar, one can use the proof of $[\mathbf{5}, \mathrm{p}$. 587-589]. In this proof, Glimm constructs a factor representation $\phi$ of $A$ which is type II (resp. III) if a certain parameter is equal to $\frac{1}{2}$ (resp. different from $\frac{1}{2}$ ). By refining this construction a little, we shall show that (under our present hypothesis) $\phi$ is actually faithful (the following idea was already used in [3]). Since $A$ is separable primitive, there exists a decreasing sequence $\left(J_{1}, J_{2}, \ldots\right)$ of nonzero closed two-sided ideals of $A$ such that every nonzero primitive ideal of $A$ contains one of the $J_{n}$ [2, Lemma 15]. If $A$ is realized as an irreducible $C^{*}$-algebra of operators in a Hilbert space, each $J_{n}$ operates irreducibly [1, 2.11.3]; granted this, we can, in the proof of [5, Lemma 4], choose the element $C_{0}$ (p. 578) in $J_{n+1}$ whence $V\left(a_{1}, \cdots, a_{n+1}\right) \in J_{n+1}$. But, coming back to p. 587 of [5], one has

$$
\begin{aligned}
&\left(\phi\left(V\left(a_{1}, \cdots, a_{n+1}\right) V\left(a_{1}, \cdots, a_{n+1}\right)^{*}\right) x \mid x\right) \\
&=h\left(V\left(a_{1}, \cdots, a_{n+1}\right) V\left(a_{1}, \cdots, a_{n+1}\right)^{*}\right) \\
&=g\left(V\left(a_{1}, \cdots, a_{n+1}\right) V\left(a_{1}, \cdots, a_{n+1}\right)^{*}\right) \neq 0,
\end{aligned}
$$


whence $V\left(a_{1}, \cdots, a_{n+1}\right) \notin \operatorname{Ker} \phi$, and so $\operatorname{Ker} \phi D J_{n+1}$. This being true for every $n$, we have $\operatorname{Ker} \phi=0$.

With $A$ antiliminar separable, Glimm constructs in [5, p. 593-596] an injective Borel map of $X / X^{\prime}$ into $\hat{A}$; with the notations of [5], $f\left(X / X^{\prime}\right)$ is equal to $K^{c}$ (or, more accurately, to the set of classes of elements of $\left.K^{c}\right)$. If $\pi \in K^{c}$, one has $\pi\left(\sum_{a_{1}, \cdots, a_{n}} V\left(a_{1}, \cdots, a_{n}\right)\right.$ $\left.V\left(a_{1}, \cdots, a_{n}\right)^{*}\right) \neq 0[5$, p. 593 , line 4 from the bottom], and so $\operatorname{Ker} \pi D J_{n}$. This proves that $\operatorname{Ker} \pi=0$, and so $f\left(X / X^{\prime}\right) \subset E$.

From this, we conclude that Card $E \geqq c$ (the power of the continuum). But it is well known that the power of the conjugate space of $A$ is $c$ so that Card $\hat{A} \leqq c$ and, hence, Card $E=c$. The proof is complete.

4. Remark. Suppose we are in case (b) of Theorem 1. Using the reasoning of [3, Corollary 4 ], one sees that there exists on $E$ standard measures which are not canonical in the sense of [4].

5. Remark. Again, let $A$ be a separable $C^{*}$-algebra. Let Prim $(A)$ be the space of primitive ideals of $A$ with the Jacobson topology. Let $p$ be the canonical map $\pi \rightarrow \operatorname{Ker} \pi$ from $\hat{A}$ onto Prim $(A)$. The Jacobson topology on $\hat{A}$ is the inverse image by $p$ of the Jacobson topology on Prim $(A)$. We shall denote by $Z_{a}$ (resp. $Z_{b}$ ) the set of all $J$ in Prim $(A)$ with the property $a$ (resp. $b$ ) of Theorem 1 . Thus, Prim $A$ is the disjoint union of $Z_{a}$ and $Z_{b}$, and $\hat{A}$ is the disjoint union of $Y_{a}$ $=p^{-1}\left(Z_{a}\right)$ and $Y_{b}=p^{-1}\left(Z_{b}\right)$. From [6, p. 22, Theorem 2] and $[1,6.9 .7]$, one sees that $Y_{a}, Y_{b}, Z_{a}, Z_{b}$ are Borel for the Jacobson topologies, and that $Y_{a}, Z_{a}$ are isomorphic standard Borel spaces. In order that a subset $Y$ of $\hat{A}$ be Borel for the Jacobson topology, it is necessary and sufficient that $Y$ be Borel for the Mackey structure, and that $Y$ $=p^{-1}(p(Y))$. From [6, p. 70, Proposition 5], we know that both $Y_{a}$ and $Y_{b}$ may be dense in $\hat{A}$.

\section{BIBLIOGRAPHY}

1. J. Dixmier, Les $C^{*}$-algèbres et leurs représentations, Gauthier-Villars, Paris, 1964.

2. - Sur les $C^{*}$-algèbres, Bull. Soc. Math. France 88 (1960), 95-112.

3. - Utilisation des facteurs hyperfinis dans la théorie des $C^{*}$-algèbres, $\mathrm{C} . \mathrm{R}$. Acad. Sci. Paris 258 (1964), 4184-4187.

4. J. Ernest, $A$ decomposition theory for unitary representations of locally compact groups, Trans. Amer. Math. Soc. 104 (1962), 252-277.

5. J. Glimm, Type I $C^{*}$-algebras, Ann. of Math. 73 (1961), 572-612.

6. A. Guichardet, Caractères des algèbres de Banach involutives, Ann. Inst. Fourier (Grenoble) 13 (1962), 1-81.

TUlane University 